PROCEEDINGS OF THE

AMERICAN MATHEMATICAL SOCIETY

Volume 136, Number 11, November 2008, Pages 3895-3903

S 0002-9939(08)09349-0

Article electronically published on May 22, 2008

\title{
SELF-SIMILAR SETS WITH AN OPEN SET CONDITION AND GREAT VARIETY OF OVERLAPS
}

\author{
CHRISTOPH BANDT AND NGUYEN VIET HUNG
}

(Communicated by Jane M. Hawkins)

\begin{abstract}
For a very simple family of self-similar sets with two pieces, we prove, using a technique of Solomyak, that the intersection of the pieces can be a Cantor set with any dimension in $[0,0.2]$ as well as a finite set of any cardinality $2^{m}$. The main point is that the open set condition is fulfilled for all these examples.
\end{abstract}

\section{INTRODUCTION}

Hutchinson 9] gave a precise mathematical framework for self-similarity, the property which was pointed out by Mandelbrot as the essence of fractals. He defined a self-similar set $A$ as the unique compact non-empty solution of an equation

$$
A=f_{1}(A) \cup \ldots \cup f_{m}(A)
$$

where $f_{1}, \ldots, f_{m}$ are given contracting similarity maps on $\mathbb{R}^{d}$, that is, $\left|f_{i}(x)-f_{i}(y)\right|=$ $r_{i} \cdot|x-y|$ for some $r_{i}<1$. Since $A$ consists of similar copies $A_{i}=f_{i}(A)$ of itself, each $A_{i}$ consists of smaller copies $A_{i j}=f_{i}\left(f_{j}(A)\right)$, and so on. For any integer $n$, we can consider the set $S^{n}$ of words $\mathbf{i}=i_{1} \ldots i_{n}$ from the alphabet $S=\{1, \ldots, m\}$. Writing $f_{\mathbf{i}}=f_{i_{1}} \ldots f_{i_{n}}$ and $A_{\mathbf{i}}=f_{\mathbf{i}}(A)$, we have $A=\bigcup\left\{A_{\mathbf{i}} \mid \mathbf{i} \in S^{n}\right\}$. When $n$ tends to infinity, this induces a continuous map $\pi: S^{\infty} \rightarrow A$ from the set $S^{\infty}$ of sequences $B=s_{1} s_{2} s_{3} \ldots$ onto the self-similar set, the so-called address map. $\pi$ is defined by

$$
\pi\left(s_{1} s_{2} s_{3} \ldots\right)=\lim _{n \rightarrow \infty} f_{s_{1}} f_{s_{2}} \ldots f_{s_{n}}\left(x_{0}\right)
$$

where the choice of $x_{0} \in \mathbb{R}^{d}$ does not matter. See [9, 7, 6] for details.

The geometric structure of $A$ is determined by the overlaps of the $A_{i}$. If the contraction factors $r_{i}$ of the $f_{i}$ are sufficiently small, the pieces $A_{i}$ are disjoint, $\pi$ is a homeomorphism and $A$ a Cantor set. This case is not interesting from a topological viewpoint. For sufficiently large $r_{i}$, however, $\pi$ identifies many addresses, and the overlaps $A_{i} \cup A_{j}$ become so big that smaller pieces $A_{\mathbf{i}}$ cannot be recognized. The equation remains formally true, but self-similarity is not realized geometrically.

The proper condition to control overlaps is the open set condition (OSC) which was introduced by Moran in 1946 and studied by Hutchinson. Several equivalent formulations of OSC are known [3, 11, 4]. Sidorov [12, gave criteria under

Received by the editors March 16, 2007, and, in revised form, September 19, 2007.

2000 Mathematics Subject Classification. Primary 28A80; Secondary 37B10, 37F20. 

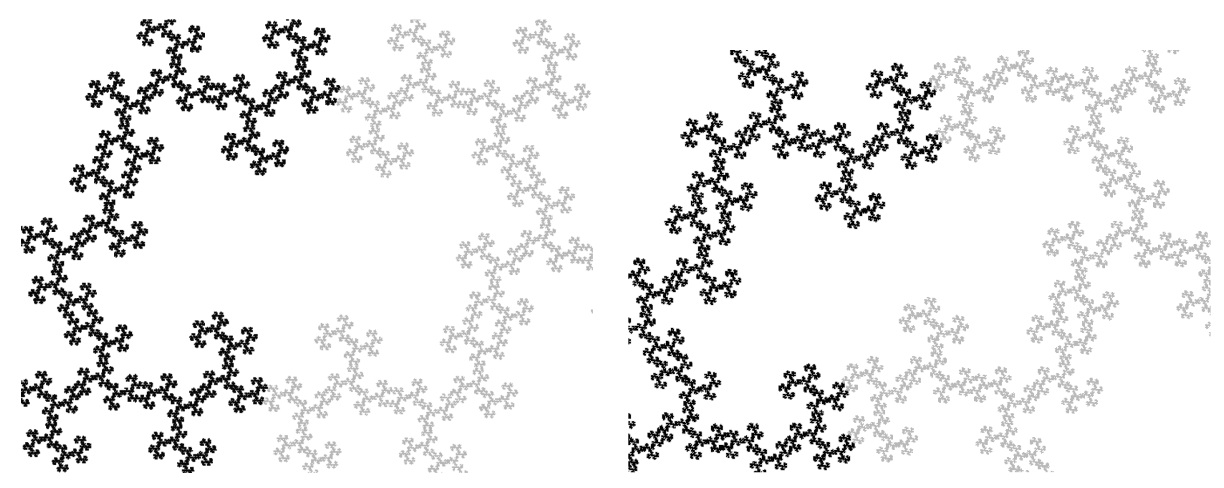

Figure 1. Self-similar sets with a Cantor set overlap of dimension around 0.2 on the left, 0.4 on the right.

which OSC fails. In general, it is difficult to verify whether a given set $f_{1}, \ldots, f_{m}$ of similarity maps fulfils OSC or not.

Actually, all examples of self-similar sets with OSC were constructed with rather special methods which either prescribe an open set (e.g. a triangle for the Sierpinski gasket and the von Koch curve) or solve equations for the mappings which in the complex plane results in mappings $f_{k}(z)=\lambda_{k} z+v_{i}$ where the $\lambda_{k}$ and $v_{k}$ are algebraic integers [1, 10. It seems that it was not known whether there are uncountably many essentially different examples with OSC.

The purpose of the present paper is to show that there is a great variety of selfsimilar sets with $O S C$. We shall consider a very simple family of fractals $A=A(\lambda)$ in the complex plane with two pieces, given by mappings

$$
f_{0}(z)=\lambda z, \quad f_{1}(z)=\lambda z+1,
$$

where $\lambda$ is a complex number with $r=|\lambda|<1$. This family was studied by several authors [6, 13, 14]. We shall only consider $\lambda$ near $0.37+0.52 i$. Let the overlap set be $A_{0} \cap A_{1}=D=D(\lambda)$. Solomyak proved the following theorem.

Theorem 1 (14], Theorem 2.4). There are uncountably many $\lambda$ for which the overlap set $D(\lambda)$ is a singleton. The two addresses of this point are different for different $\lambda$.

Together with the statement in [5] that connected self-similar sets in the plane with finite overlap sets fulfil OSC, this provides uncountably many different examples with OSC. In the present note we use Solomyak's technique to give examples with various other overlap sets. Figure 1 shows the type of fractals we shall deal with.

Theorem 2. For every $m \in \mathbb{N}$ there are uncountably many $\lambda$ for which OSC holds, and the overlap set $D(\lambda)$ consists of $2^{m}$ points. The addresses of these points are different for different $\lambda$.

Theorem 3. For every $\beta \in[0,0.2]$ there are uncountably many $\lambda$ for which OSC holds, and the overlap set $D(\lambda)$ is a Cantor set of Hausdorff dimension $\beta$. The addresses of the points of $D(\lambda)$ are different for different $\lambda$. 


\section{The STRUCtURE OF OVERLAP}

We use the symbol set $S=\{0,1\}$ for our mappings (2) and we now start addresses $ß$ with $s_{0}$ since this simplifies the address map (1) with $x_{0}=0$ :

$$
\pi(ß)=\pi\left(s_{0} s_{1} s_{2} \ldots\right)=\sum_{k=0}^{\infty} s_{k} \lambda^{k} .
$$

It is easy to check that $\pi$ is one-to-one if $A_{0} \cap A_{1}=\emptyset$, and $A$ is connected if $D(\lambda)=A_{0} \cap A_{1} \neq \emptyset[8$, 6]. To represent the overlap set, take two sequences $\beta=0 s_{1} s_{2} \ldots$ and $\mathbf{t}=1 t_{1} t_{2} \ldots$ with $\pi(ß)=\pi(\mathbf{t})$. This means

$$
\pi(\mathbf{t})-\pi(ß)=1+\sum_{k=1}^{\infty}\left(t_{k}-s_{k}\right) \lambda^{k}=0 .
$$

Note that $t_{k}-s_{k} \in\{-1,0,1\}$. Moreover, for any $u_{k} \in\{-1,0,1\}$ there are $t_{k}, s_{k} \in$ $\{0,1\}$ with $u_{k}=t_{k}-s_{k}$. Thus

Remark 1. $D(\lambda) \neq \emptyset$ if and only if $\lambda$ is a root of a power series with coefficients $-1,0,1$.

When $\lambda$ is such a root, we can transform the power series into the form

$$
f(\lambda)=1+\sum_{k=1}^{\infty} u_{k} \lambda^{k}, \quad u_{k} \in\{-1,0,1\}
$$

and then choose $t_{k}$ and $s_{k}$. If $u_{k}=1$, then $t_{k}=1, s_{k}=0$. If $u_{k}=-1$, then $t_{k}=0, s_{k}=1$. However, if $u_{k}=0$, then we have two choices: $t_{k}=s_{k}=0$ or $t_{k}=s_{k}=1$.

Notation. We write $\mathbf{u}=1 u_{1} u_{2} \ldots$ where $u_{k_{j}}=0$ for $j=1,2, \ldots$ In other words, $\mathbf{u}=1 w_{1} 0 w_{2} 0 w_{3} 0 \ldots$, where the $w_{j} \in\{-1,1\}^{*}$ are words made from -1 and $+1\left(w_{j}\right.$ can be empty), and zeros appear at places $k_{1}, k_{2}, \ldots$.

If 0 appears at $m$ different places $k_{1}, \ldots, k_{m}$, then we can choose 0 or 1 for each $s_{k_{j}}$ independently, and we get $2^{m}$ possible pairs of addresses $\beta$, t with $\pi(\beta)=\pi(\mathbf{t})$.

Remark 2. Suppose that $\lambda$ is the root of exactly one power series of the form (5) with $u_{k} \in\{-1,0,1\}$. Then $D(\lambda)$ is a singleton if no coefficient is 0 , and $D(\lambda)$ has $2^{m}$ points if just $m$ of the coefficients $u_{k}$ are zero. If $u_{k}=0$ for infinitely many $k=k_{1}, k_{2}, \ldots$ and $\left|k_{n+1}-k_{n}\right|>2$ for all sufficiently large $n$, then $D(\lambda)$ is a Cantor set.

Proof. It remains to verify the last statement. For each $w_{j}$ in $\mathbf{u}$ there is a word $\tilde{w}_{j} \in\{0,1\}^{*}$ which contains the corresponding part of addresses $\beta$ associated with $\mathbf{u}$, that is, $\tilde{w}_{j i}=1$ if $w_{j i}=-1$ and $\tilde{w}_{j i}=0$ for $w_{j i}=1$. Thus

$$
D=D(\lambda)=\left\{\pi(\beta) \mid \beta=0 \tilde{w}_{1} v_{1} \tilde{w}_{2} v_{2} \tilde{w}_{3} v_{3} \ldots \text { with } v_{j} \in\{0,1\}\right\}
$$

indicates a binary Cantor structure: $D=D_{0 \tilde{w}_{1} 0} \cup D_{0 \tilde{w}_{1} 1}$, and $D_{0 \tilde{w}_{1} 0}=D_{0 \tilde{w}_{1} 0 \tilde{w}_{2} 0} \cup$ $D_{0 \tilde{w}_{1} 0 \tilde{w}_{2} 1}$, etc., similar as for the whole set $A$. Since (1) holds for $\pi(ß)$, each piece $D_{\tilde{w}}$ is a subset of $A_{\tilde{w}}$.

Is $D$ really a Cantor set? For $\lambda=i / \sqrt{2}$ the set $A$ is a rectangle and $D$ an interval. Thus we have to show that the two subpieces of each piece of $D$ are disjoint. Assume that on the $n$-th level of $D$ there is some $x \in D_{\tilde{w} 0} \cap D_{\tilde{w} 1}$. Here $\tilde{w}=0 \tilde{w}_{1} v_{1} \tilde{w}_{2} v_{2} \ldots \tilde{w}_{n}$ 
has length $k_{n}$. Now $x$ has two addresses, $\beta=\tilde{w} 1 \tilde{w}_{n+1} v_{n+1} \tilde{w}_{n+2} v_{n+2} \ldots$ and $\beta^{\prime}=$ $\tilde{w} 0 \tilde{w}_{n+1} v_{n+1}^{\prime} \tilde{w}_{n+2} v_{n+2}^{\prime} \ldots$, so that

$0=\pi(B)-\pi\left(B^{\prime}\right)=\lambda^{k_{n}}+\sum_{m=n+1}^{\infty}\left(v_{m}-v_{m}^{\prime}\right) \lambda^{k_{m}}=\lambda^{k_{n}}\left(1+\sum_{m=n+1}^{\infty}\left(v_{m}-v_{m}^{\prime}\right) \lambda^{k_{m}-k_{n}}\right)$.

Since the coefficients $u_{k}$ of the power series (5) for $\lambda$ are unique, this implies that $u_{k} \neq 0$ is only possible for $k=k_{m}-k_{n}, m=n+1, n+2, \ldots$. Thus by our assumption the non-zero elements in $\mathbf{u}$ have distance $>2$ for sufficiently large $k$. Thus there are many neighboring zeros, which contradicts the same assumption and completes the proof.

We think the assumption on distance $>2$ was not necessary. In the following we estimate the Hausdorff dimension of the Cantor set $D$ in terms of density of the sequence $k_{n}$.

Notation. Let $\mathcal{H}^{\alpha}$ denote the $\alpha$-dimensional Hausdorff measure [7, let $a=|A|$ be the diameter of $A$, and let $r=|\lambda|$ be the contraction factor of our mappings $f_{0}, f_{1}$.

Remark 3. If $r^{k_{n} \gamma} \leq c \cdot 2^{-n}$ for a constant $c$ and infinitely many $n$, then $\mathcal{H}^{\gamma}(D)<\infty$.

Proof. Take $n$ which fulfills the inequality, and consider the $2^{n}$ initial words $\beta^{\prime}$ of length $k_{n}$ of sequences $\beta$ which correspond to $\mathbf{u}$. Then $D$ is covered by the $2^{n}$ sets $A_{\beta^{\prime}}$ which all have diameter $r^{k_{n}} a$. The sum for this covering in the definition of $\mathcal{H}^{\gamma}(D)$ is

$$
2^{n} \cdot\left(r^{k_{n}} a\right)^{\gamma} \leq 2^{n} \cdot c \cdot 2^{-n} a^{\gamma} \leq c a^{\gamma} .
$$

If this holds for arbitrary large $n$, then $\mathcal{H}^{\gamma}(D) \leq c a^{\gamma}$.

Remark 4. Assume that OSC holds for the set $A$ generated by $f_{0}, f_{1}$, and that the zeros in $\mathbf{u}$ have bounded gaps: $k_{n+1}-k_{n}<K$ for all $n$. If $r^{k_{n} \alpha} \geq c \cdot 2^{-n}$ for a constant $c$ and all $n \geq n^{*}$, then $\mathcal{H}^{\alpha}(D)>0$.

Proof. Let $\mu$ denote the binary construction measure on $D$, considered as a measure on $\mathbb{R}^{2}$. That is, $\mu(D)=1$ and $\mu\left(D_{w}\right)=2^{-n}$ for each of the $2^{n}$ pieces $D_{w}$ of $D$ which are obtained on the level $n$ of the construction, which corresponds to $u_{k_{n}}$.

OSC for $A$ implies that there is a constant $L$ such that each set $B$ of diameter $b=|B|$ hits at most $L$ incomparable pieces $A_{w}$ of diameter $\geq b \cdot r^{K}$ [11, 4]. (Instead of $r^{K}$ we can have any constant, and incomparable means we do not allow words $w, w^{\prime}$ where $w^{\prime}$ is an initial subword of $w$.) For $r^{k_{n}}<b<r^{k_{n-1}}$ with $n \geq n^{*}$ this implies

$$
\mu(B) \leq 2^{-n} \cdot \operatorname{card}\left\{A_{w}\left|A_{w} \cap B \neq \emptyset,\right| w \mid=k_{n}\right\} \leq L 2^{-n} \leq \frac{L}{c} r^{k_{n} \alpha} \leq \frac{L}{c}|B|^{\alpha} .
$$

Now take a covering $D \subseteq \bigcup B_{i}$ where the diameter of each $B_{i}$ is smaller than $r^{k_{n^{*}}}$. Then

$$
\sum\left|B_{i}\right|^{\alpha} \geq \frac{c}{L} \sum \mu\left(B_{i}\right) \geq \frac{c}{L} .
$$

This implies $\mathcal{H}^{\alpha}(D) \geq \frac{c}{L}$. 


\section{The MAIN LEMma}

The parameters $\lambda$ in our theorems will be defined as roots of power series (5) with special coefficient vectors $\mathbf{u}=u_{0} u_{1} \ldots$. The assignment $u \mapsto \lambda$ has a certain continuity property:

Remark 5. Let $f$ be a power series of the form (5), $\lambda_{0}$ a root of $f$ and $G_{0}$ a simply connected neighborhood of $\lambda_{0}$. There is a $k$ such that every power series $g$ of the form (5) with coefficients $v_{0} v_{1} v_{2} \ldots$ such that $v_{j}=u_{j}$ for $j \leq k$ has a root in $G_{0}$.

Proof with Rouchés theorem. If $f, h$ are analytic functions on the closure of a simply connected domain $G$, and for all points $z$ of the boundary $\partial G$ we have $f(z) \neq 0$ and $|h(z)|<|f(z)|$, then $f$ and $f+h$ have the same number of zeros in $\mathrm{G}$.

Since the zeros of $f$ are discrete, we can find a ball $G \subseteq G_{0}$ around $\lambda_{0}$ such that $f(z) \neq 0$ on $G \backslash\left\{\lambda_{0}\right\}$. Let the minimum of $|f(z)|$ on the boundary $\partial G$ be $\varepsilon$, the radius of the ball $\delta$ and $\eta=\left|\lambda_{0}\right|+\delta$. Choose $k$ so that $\eta^{k}(1-\eta)^{-1}<\varepsilon$, let $h=g-f$ and apply Rouché's theorem. In $G$ every $g$ has exactly one root.

Now we approach the parameters $\lambda$ which will fulfill our theorems. The set

$$
\Lambda=\left\{\lambda \in \mathbb{C}|0.63<| \lambda \mid<0.64 \text { and } 54^{\circ}<\arg (\lambda)<55^{\circ}\right\}
$$

contains $\lambda^{*} \approx 0.3668760+0.5202594 i$, which is a zero of the power series $f^{*}(\lambda)$ with

$$
\mathbf{u}^{*}=u_{0}^{*} u_{1}^{*} u_{2}^{*} \ldots=+-(+++---)^{\infty} .
$$

Here we write ' + ' for 1 and ' - ' for -1 , and $(+++---)^{\infty}=+++---+++$ $---\ldots$. Direct calculation shows that $\lambda^{*}$ is a solution of $1-\lambda+\lambda^{2}+2 \lambda^{3}=0$; see Example 9.4 in 2]. Solomyak proved Theorem 1 by slightly changing the parameter $\lambda^{*}$.

According to Remark 5, there is a minimal $m_{0}$ such that every power series (5) with coefficients starting with $+-(+++---)^{m_{0}}$ has a zero in $\Lambda$. Actually $m_{0}$ is not very large: Solomyak notes that he can prove $m_{0} \leq 14$, and computer experiments indicate that $m_{0}=2$ [14]. We define

$$
\begin{aligned}
& \mathcal{B}=\left\{g(z)=1+\sum_{j=1}^{\infty} v_{j} z^{j} \mid v_{j}=u_{j} \text { for } j \leq 6 m_{0}+1,\right. \\
& \text { and } v_{2} v_{3} \ldots \text { is a concatenation of blocks }+++---,++++---, \\
& \quad+++----,++++----,+++0---\} .
\end{aligned}
$$

For power series $f, g$ of the form (5), the term $R_{g-f}$ will denote a normalized difference: if $g(z)-f(z)=z^{l} \sum_{j=0}^{\infty} c_{j} z^{j}$ with $c_{0} \neq 0, l \geq 1$ and $c_{j} \in\{-2,-1,0,1,2\}$, let

$$
R_{g-f}(z)=\frac{g(z)-f(z)}{z^{l}}=\sum_{j=0}^{\infty} c_{j} z^{j} .
$$

The following lemma and its proof refine a technique of Solomyak. See Section 4 in [14.

Lemma 1. For all power series $f \neq g$ of the form (5) with $g \in \mathcal{B}$ and all $\lambda \in \Lambda$ we have

$$
\left|R_{g-f}(\lambda)\right|>10^{-3}
$$


Proof. With $l$ defined as above, we consider an exhaustive list of possibilities for the coefficients $v_{l}, v_{l+1}, \ldots$ of $g \in \mathcal{B}$ :

$$
\begin{aligned}
& \text { (i) } \quad\left[v_{l}, v_{l+5}\right]=++++--\quad\left(i^{\prime}\right) \quad\left[v_{l}, v_{l+5}\right]=---++ \\
& \text { (ii) }\left[v_{l}, v_{l+4}\right]=+++--\quad\left(i i^{\prime}\right) \quad\left[v_{l}, v_{l+4}\right]=---++
\end{aligned}
$$

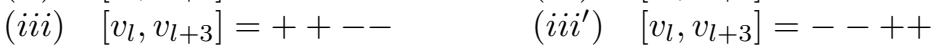

$$
\begin{aligned}
& \text { (iv) }\left[v_{l}, v_{l+3}\right]=+---\quad\left(i v^{\prime}\right) \quad\left[v_{l}, v_{l+3}\right]=-+++ \\
& \text { (v) } \quad\left[v_{l}, v_{l+5}\right]=+++0-- \\
& \text { (vi) } \quad\left[v_{l}, v_{l+4}\right]=++0-- \\
& \text { (vii) } \quad\left[v_{l}, v_{l+4}\right]=+0--- \\
& \text { (viii) }\left[v_{l}, v_{l+5}\right]=0---++
\end{aligned}
$$

We prove $\left|R_{g-f}(\lambda)\right|>10^{-3}$ by first examining the cases $(i)-(i v)$. Then $\left(i^{\prime}\right)-$ $\left(i v^{\prime}\right)$ will follow by passing from $R_{g-f}(\lambda)$ to $-R_{g-f}(\lambda)$, and after that we study the remaining cases.

Case (i). From the values of $v_{l}, \ldots, v_{l+5}$, we derive that $c_{0} \in\{1,2\}, c_{1}, c_{2}, c_{3} \in$ $\{0,1,2\}$, and $c_{4}, c_{5} \in\{0,-1,-2\}$. We can estimate as follows:

$$
\left\{\begin{array}{l}
\operatorname{Re}\left(c_{0} \lambda^{-1}\right) \geq|\lambda|^{-1} \cos \left(55^{\circ}\right)>0.896 \\
\operatorname{Re}\left(c_{1}\right), \operatorname{Re}\left(c_{2} \lambda\right), \operatorname{Re}\left(c_{4} \lambda^{3}\right), \operatorname{Re}\left(c_{5} \lambda^{4}\right) \geq 0 \\
\operatorname{Re}\left(c_{3} \lambda^{2}\right) \geq 2|\lambda|^{2} \cos \left(110^{\circ}\right)>-0.281 \\
\operatorname{Re}\left(\sum_{j=6}^{\infty} c_{j} \lambda^{j-1}\right) \geq-\frac{2 \cdot|\lambda|^{5}}{1-|\lambda|}>-0.597
\end{array}\right.
$$

Taking the sum, we get $\operatorname{Re}\left(\frac{R_{g-f}(\lambda)}{\lambda}\right)>0.002$, and

$$
\left|R_{g-f}(f)(\lambda)\right|=\left|\frac{R_{g-f}(\lambda)}{\lambda}\right||\lambda| \geq \operatorname{Re}\left(\frac{R_{g-f}(\lambda)}{\lambda}\right)|\lambda|>0.002 \cdot 0.5=10^{-3} .
$$

Case (ii). We have $c_{0} \in\{1,2\}, c_{1}, c_{2} \in\{0,1,2\}$, and $c_{3}, c_{4} \in\{0,-1,-2\}$. Hence,

$$
\left\{\begin{array}{l}
\operatorname{Re}\left(c_{0}\right) \geq 1 \\
\operatorname{Re}\left(c_{1} \lambda\right), \operatorname{Re}\left(c_{3} \lambda^{3}\right), \operatorname{Re}\left(c_{4} \lambda^{4}\right) \geq 0 \\
\operatorname{Re}\left(c_{2} \lambda^{2}\right) \geq 2|\lambda|^{2} \cos \left(110^{\circ}\right)>-0.281 \\
\operatorname{Re}\left(\sum_{j=5}^{\infty} c_{j} \lambda^{j}\right) \geq-\frac{2 \cdot|\lambda|^{5}}{1-|\lambda|}>-0.597
\end{array}\right.
$$

These inequalities lead to $\operatorname{Re}\left(R_{g-f}(\lambda)\right)>10^{-3}$. It follows that $\left|R_{g-f}(\lambda)\right|>10^{-3}$.

Case (iii). We have $c_{0} \in\{1,2\}, c_{1} \in\{0,1,2\}, c_{2}, c_{3} \in\{0,-1,-2\}$. Thus,

$$
\left\{\begin{array}{l}
\operatorname{Re}\left(c_{0}\right) \geq 1, \\
\operatorname{Re}\left(c_{1} \lambda\right), \operatorname{Re}\left(c_{2} \lambda^{2}\right), \operatorname{Re}\left(c_{3} \lambda^{3}\right) \geq 0, \\
\operatorname{Re}\left(\sum_{j=4}^{\infty} c_{j} \lambda^{j}\right) \geq-\frac{2 \cdot|\lambda|^{4}}{1-|\lambda|}>-0.933
\end{array}\right.
$$

These inequalities also lead to $\operatorname{Re}\left(R_{g-f}(\lambda)\right)>10^{-3}$.

Case (iv). We have $c_{0} \in\{1,2\}, c_{1}, c_{2}, c_{3} \in\{0,-1,-2\}$. Note that in this case, $v_{l+5}=1$, and hence $c_{5} \in\{0,1,2\}$. So,

$$
\left\{\begin{array}{l}
\operatorname{Im}\left(-c_{0} \lambda^{-1}\right) \geq \operatorname{Im}(\lambda) /|\lambda|^{2}>1, \\
\operatorname{Im}\left(-c_{1}\right), \operatorname{Im}\left(-c_{2} \lambda\right), \operatorname{Im}\left(-c_{3} \lambda^{2}\right), \operatorname{Im}\left(-c_{5} \lambda^{4}\right) \geq 0, \\
\operatorname{Im}\left(-c_{4} \lambda^{3}\right) \geq-2|\lambda|^{3} \sin \left(162^{\circ}\right)>-0.163, \\
\operatorname{Im}\left(\sum_{j=5}^{\infty}-c_{j+1} \lambda^{j}\right) \geq-\frac{2 \cdot||^{5}}{1-|\lambda|}>-0.6 .
\end{array}\right.
$$

It follows that $\operatorname{Im}\left(\frac{-R_{g-f}(\lambda)}{\lambda}\right)>0.002$ and $\left|R_{g-f}(\lambda)\right| \geq 0.002 \cdot 0.5$ as in $(i)$. 
Case (v). Note that $c_{3} \in\{1,0,-1\}$. If $c_{3} \in\{0,1\}$, we can use the estimate of $(i)$, while $c_{3}=-1$ leads to case $(i i)$.

Case (vi). We have $c_{2} \in\{1,0,-1\}$. If $c_{2} \in\{0,1\}$, we proceed as in case (ii). Case $c_{2}=-1$ leads to case $($ iii).

Case (vii). $c_{1} \in\{0,1\}$ is treated like (iii), and $c_{1}=-1$ like (iv).

Case (viii). $c_{0}=1$ leads to $(i v)$ and $c_{0}=-1$ to $\left(i^{\prime}\right)$.

\section{Proof of the theorems}

Remark 6. If $g \in \mathcal{B}$ and $\lambda \in \Lambda$ fulfills $g(\lambda)=0$, then $\lambda$ is not a root of any other power series $f$ of the form (5).

Proof. $f(\lambda)=0$ would imply $R_{g-f}(\lambda)=0$, which contradicts our lemma.

Remark 7. If $g \in \mathcal{B}$ and $\lambda \in \Lambda$ is a root of $g$, then $A(\lambda)$ satisfies the open set condition.

Proof. According to 3], OSC holds if and only if the identity map is not an accumulation point of the neighbor maps $f_{v}^{-1} f_{w}$ where $v=\left(v_{1} \ldots v_{m}\right), w=\left(w_{1} \ldots w_{m}\right) \in$ $\{0,1\}^{m}$ with $v_{1} \neq w_{1}$ and $m \in \mathbb{N}$. In our case, these maps are all translations (cf. [3], Section 2):

$$
f_{v}^{-1} f_{w}(z)=z+\sum_{j=1}^{m} \frac{1}{\lambda^{j}} \cdot\left(w_{j}-v_{j}\right)=z+\frac{p(\lambda)}{\lambda^{m}}
$$

where $p(\lambda)=\sum_{j=0}^{m-1} b_{j} \lambda^{j}$ is a polynomial with coefficients $b_{j} \in\{-1,0,1\}$. We need to show that the translation vectors are bounded away from zero, uniformly in $p$. We can assume that $b_{0}=1$. Since the coefficient of $g(\lambda)$ at either $\lambda^{m}$ or $\lambda^{m+1}$ is non-zero, the lemma gives

$$
\left|\frac{p(\lambda)}{\lambda^{m}}\right|=\left|\frac{g(\lambda)-p(\lambda)}{\lambda^{m+1}}\right| \cdot|\lambda| \geq\left|R_{g-p}(\lambda)\right| \cdot|\lambda|>\frac{1}{2} \cdot 10^{-3} .
$$

Proof of Theorem 2. We consider all $g \in \mathcal{B}$ with exactly $m$ coefficients 0 . By Remark 5 , to each $g$ we can take a root $\lambda \in \Lambda$. From the definition of $\mathcal{B}$ it is clear that there are uncountably many possibilities and that the addresses of points in $D(\lambda)$ will be different for different $\lambda$. Remark 2 says that $D(\lambda)$ has $2^{m}$ points. OSC follows from Remark 7 or from [5].

Proof of Theorem 3 . We take the $g \in \mathcal{B}$ with infinitely many coefficients 0 . For simplicity, we consider only coefficient vectors $+-w_{1} w_{2} \ldots$ where each block $w_{j}$ is either +++--- or $+++0---$. By Remark 6 , to each $g$ we can take a root $\lambda \in \Lambda$. Then $D(\lambda)$ is a Cantor set by Remark 2, and OSC holds by Remark 7. Thus the dimension of $A(\lambda)$ equals $\delta=\frac{\log 2}{-\log r} \geq \frac{\log 2}{-\log 0.63}>1.5$ where $r=|\lambda|$. In other words, $r=2^{-1 / \delta}$.

Let $\beta$ be given. Remarks 3 and 4 say that $\operatorname{dim} D(\lambda)=\beta$ if $r^{k_{n} \beta}=2^{-k_{n} \beta / \delta}$ is of the same order as $2^{-n}$. This means $k_{n} \approx \frac{\delta}{\beta} \cdot n$ and will be realized by choosing the zero coefficients, i.e. by selecting the $w_{j}$. Since $k_{n+1}-k_{n} \geq 7$, this requires that $\beta<\delta / 7$. (With modified blocks including +++0 and ---0 one can obtain $\beta$ up to $\delta / 4$. By the way, periodic patterns of the $k_{n}$ yield self-similar sets $D(\lambda)$.)

Since $r$ is part of the construction, however, we must determine $r$ and the $k_{n}$ recursively. We define a decreasing sequence of closed balls $G_{l}=B_{\eta_{l}}\left(\lambda_{l}\right)$ where $\eta_{l} \leq \eta_{l-1} / 3$, which will imply the existence of the limit $\lambda=\lim \lambda_{l}=\bigcap G_{l}$. We start 
with $\lambda_{0}=\lambda^{*}$ and $\eta_{0}=0.03$ so that $G_{0} \subseteq \Lambda$ since $r_{0} \approx 0.6366$. For $\varepsilon=0.02$ we have $\frac{r_{0}+\eta_{0}}{r_{0}-\eta_{0}} \leq 0.64^{2 \varepsilon}$, which implies $\left(r_{0}+\eta_{0}\right)^{\beta(1+\varepsilon)} \leq\left(r_{0}-\eta_{0}\right)^{\beta(1-\varepsilon)}$ for any $\beta$. Moreover, $\left(\frac{r_{l}+\eta_{l}}{r_{l}-\eta_{l}}\right)^{2}<\frac{r_{l-1}+\eta_{l-1}}{r_{l-1}-\eta_{l-1}}$ for $l \geq 1$. (Put $x=\frac{\eta_{l}}{r_{l}}, y=\frac{\eta_{l-1}}{r_{l-1}}$; then $x<\frac{1}{3} \frac{0.64}{0.63} y<0.35 y<$ 0.02 implies $(1+x)^{2}<1+y$ and $(1-x)^{2}>1-y$.) Thus

$$
\left(r_{l}+\eta_{l}\right)^{\beta\left(1+\varepsilon / 2^{l}\right)} \leq\left(r_{l}-\eta_{l}\right)^{\beta\left(1-\varepsilon / 2^{l}\right)} \quad \text { for any } \beta \quad \text { and } l=0,1,2, \ldots
$$

We want to show

$$
\text { (8) } \beta-\varepsilon / 2^{l-1} \leq \operatorname{dim} A\left(\lambda_{l}\right) \leq \beta+\varepsilon / 2^{l-1} \quad \text { for } l=1,2 \ldots, \quad \text { and } \operatorname{dim} A(\lambda)=\beta \text {. }
$$

Each $\lambda_{l}$ will be a root of a power series $g_{l} \in \mathcal{B}$ with coefficient vector $\mathbf{v}_{l}$ of the form $+-w_{1} w_{2} \ldots$. We shall construct an increasing sequence $m_{0}<m_{1}<m_{2} \ldots$ such that the first $m_{l}$ blocks $w_{j}$ are the same for all $\mathbf{v}_{k}$ with $k \geq l$. The number $m_{0}$ corresponding to $\mathbf{v}_{\mathbf{0}}=+-(+++---)^{\infty}$ was defined in Section 3, and we have $w_{1}=\ldots=w_{m_{0}}=+++---$.

Let us define $\mathbf{v}_{1}$. We take $+++0---=w_{m_{0}+1}=w_{m_{0}+2}=\ldots$ and note the positions $k_{1}, k_{2}$ of the zeros until for some $n=n_{0}$, the inequality

$$
\left(r_{0}-\eta_{0}\right)^{k_{n} \beta(1-\varepsilon)} \geq 2^{-n}
$$

is fulfilled for the first time. Here is the point where we use $\beta \leq 0.2$ : For large $n$ we have $k_{n}<7.1 n$, and $0.63^{(0.2 \cdot 7.1)}>\frac{1}{2}$. All $k_{n}$ for $n>n_{0}$ are then chosen as large as possible so that (9) remains true. From this maximality and (7) it follows that

$$
\left(r_{0}+\eta_{0}\right)^{k_{n} \beta(1+\varepsilon)} \leq c 2^{-n} \quad \text { with } c=0.64^{6 \beta} \geq\left(r_{0}-\eta_{0}\right)^{7 \beta(1-\varepsilon)} .
$$

This way we obtain the sequence $\mathbf{v}_{1}$, and its power series $g_{1} \in \mathcal{B}$ with root $\lambda_{1} \in G_{0}$ such that (8) holds for $l=1$, by Remarks 3 and 4 .

Now we specify our choice of $G_{1}=B_{\eta_{1}}\left(\lambda_{1}\right)$. Besides $\eta_{1} \leq \eta_{0} / 3$ and $G_{1} \subset G_{0}$ we require that the power series $g_{1}$ associated with $\mathbf{v}_{1}$ has no other zero in the closure of $G_{1}$. Next, $m_{1}$ is chosen so that each power series $g \in \mathcal{B}$ which coincides with $\mathbf{v}_{1}=+-w_{1} w_{2} \ldots$ in the blocks $w_{1}, \ldots, w_{m_{1}}$ must have exactly one zero in $G_{1}$. Those blocks are kept for all $\mathbf{v}_{k}, k \geq 1$.

The $k_{n}$ which are beyond $w_{m_{1}}$ are now redefined for $\mathbf{v}_{2}$. If necessary, we first take blocks $+++0---$ until for some $n=n_{1}$, the inequality $\left(r_{1}-\eta_{1}\right)^{k_{n} \beta(1-\varepsilon / 2)} \geq 2^{-n}$ is fulfilled for the first time. All $k_{n}$ for $n>n_{1}$ are then chosen as large as possible so that the inequality remains true. With (7) we have $\left(r_{1}+\eta_{1}\right)^{k_{n} \beta(1+\varepsilon / 2)} \leq c 2^{-n}$ for $n>n_{1}$, and we obtain $\mathbf{v}_{2}, g_{2} \in \mathcal{B}$ and $\lambda_{2} \in G_{1}$ such that (8) holds for $l=2$.

Induction gives $\mathbf{v}_{l}, \lambda_{l}, n_{l}$ for $l \geq 1$. The sequence $\mathbf{v}=\lim \mathbf{v}_{l}$ corresponds to $\lambda=\lim \lambda_{l}$. The $k_{n}$ in $\mathbf{v}$ holds for all

$\left(r_{l}-\eta_{l}\right)^{k_{n} \beta\left(1-\varepsilon / 2^{l}\right)} \geq 2^{-n} \quad$ and $\quad\left(r_{l}+\eta_{l}\right)^{k_{n} \beta\left(1+\varepsilon / 2^{l}\right)} \leq c 2^{-n} \quad$ for $n_{l} \leq n<n_{l+1}$.

Since $\lambda \in G_{l}$, the factor $r=|\lambda|$ lies between $r_{l}-\eta_{l}$ and $r_{l}+\eta_{l}$, the min and max of $|z|$ in $G_{l}$, for all $l$. Thus

$$
r^{k_{n} \beta\left(1-\varepsilon / 2^{l}\right)} \geq 2^{-n} \quad \text { and } \quad r^{k_{n} \beta\left(1+\varepsilon / 2^{l}\right)} \leq c 2^{-n} \quad \text { for all } n \geq n_{l} .
$$

By Remarks 3 and $4, \beta\left(1-\varepsilon / 2^{l}\right) \leq \operatorname{dim} A(\lambda) \leq \beta\left(1+\varepsilon / 2^{l}\right)$. This holds for all $l$; thus $\operatorname{dim} A(\lambda)=\beta$.

In $\mathbf{v}$ there are infinitely many pairs of different blocks $w_{j}, w_{j+1}$. Exchanging those two blocks for any set of the pairs gives a new sequence $\mathbf{v}^{\prime}$ but does not change the 
dimension since every $k_{n}$ changes by at most \pm 7 . So there are uncountably many ways to obtain dimension $\beta$.

\section{ACKNOWLEDGEMENTS}

This work was done when the second author visited the University of Greifswald, supported by the Ministry of Education and Training of Vietnam and by the DAAD.

\section{REFERENCES}

[1] C. Bandt, Self-similar measures, Ergodic Theory, Analysis and Efficient Simulation of Dynamical Systems (ed. B. Fiedler), Springer, 2001, 31-46. MR 1850300(2002j:28011)

[2] C. Bandt, On the Mandelbrot set for pairs of linear maps, Nonlinearity 15 (2002), 1127-1147. MR.1912290 (2004f:28010)

[3] C. Bandt and S. Graf, Self-similar sets VII. A characterization of self-similar fractals with positive Hausdorff measure. Proc. Amer. Math. Soc. 114 (1992), 995-1001. MR1100644 (93d:28014)

[4] C. Bandt, N.V. Hung and H. Rao, On the open set condition for self-similar fractals, Proc. Amer. Math. Soc. 134 (2005), 1369-1374. MR2199182 (2006m:28007)

[5] C. Bandt and H. Rao, Topology and separation of self-similar fractals in the plane, Nonlinearity 20 (2007), 1463-1474. MR2327133

[6] M.F. Barnsley, Fractals Everywhere, 2nd ed., Academic Press, 1993. MR.1231795|(94h:58101)

[7] K.J. Falconer, Fractal Geometry, Wiley, 1990. MR1102677(92j:28008)

[8] M. Hata, On the structure of self-similar sets, Japan J. Appl. Math. 2 (1985), 281-414. MR839336 (87g:58080)

[9] J.E. Hutchinson, Fractals and self-similarity, Indiana Univ. Math. J. 30 (1981), 713-747. MR625600 (82h:49026)

[10] S.M. Ngai and Y. Wang, Hausdorff dimension of overlapping self-similar sets. J. London Math. Soc. 63 (2001), 655-672. MR1825981 (2002c:28010)

[11] A. Schief, Separation properties for self-similar sets. Proc. Amer. Math. Soc. 122 (1994), 111-115. MR.1191872 (94k:28012)

[12] N. Sidorov, Combinatorics of linear iterated function systems with overlaps, Nonlinearity 20 (2007), 1290-1312. MR2312394

[13] B. Solomyak, 'Mandelbrot set' for pairs of linear maps: the local geometry, Analysis in Theory and Applications 20:2 (2004), 149-157. MR2095457(2005i:28022)

[14] B. Solomyak, On the 'Mandelbrot set' for pairs of linear maps: asymptotic self-similarity, Nonlinearity 18 (2005), 1927-1943. MR2164725 (2006d:37086)

Institute for Mathematics and Informatics, Arndt University, 17487 Greifswald, GERMANY

E-mail address: bandt@uni-greifswald.de

Department of Mathematics, Hue University, Hue, Vietnam

E-mail address: nvh0@yahoo.com 